Portland State University

PDXScholar

\title{
Creating Clinically Useful In Silico Models of Intracranial Pressure Dynamics
}

\author{
Wayne W. Wakeland \\ Portland State University, wakeland@pdx.edu \\ Joe Fusion \\ Portland State University \\ Brahm Goldstein \\ Oregon Health \& Science University
}

Follow this and additional works at: https://pdxscholar.library.pdx.edu/sysc_fac

Part of the Biomedical Commons

Let us know how access to this document benefits you.

\section{Citation Details}

Wakeland, W., Fusion, J., and Goldstein, B. Creating Clinically Useful in silico Models of Intracranial Pressure Dynamics. Poster presentation at the 4th International Conference on Complexity in Acute Illness (ICCAI), Cologne, 2005.

This Poster is brought to you for free and open access. It has been accepted for inclusion in Systems Science Faculty Publications and Presentations by an authorized administrator of PDXScholar. Please contact us if we can make this document more accessible: pdxscholar@pdx.edu. 


\section{Creating Clinically Useful in silico Models of Intracranial Pressure Dynamics}

Wayne Wakeland ${ }^{1}$, Joe Fusion ${ }^{1}$, Brahm Goldstein ${ }^{2}$

Aim

${ }^{1}$ System Science Ph.D. Program, Portland State University, Portland, Oregon USA

2 Oregon Health \& Science University, Portland, Oregon USA

To create clinically useful computer simulation models of intracranial pressure (ICP) dynamics by using prospective clinical data to estimate subject-specific physiologic parameters.

\section{Methods}

- A differential equation-based model was used to simulate ICP dynamics - ICP data was collected from patients with severe traumatic brain injury - During data collection, changes to Head of Bed (HOB) and Respiratory Rate (RR) were applied according to a physiological challenge protocol

- Patient-specific parameters were estimated by a curve-fitting algorithm to minimize error between the model's prediction and the recorded ICP

- Estimated parameters included: base cranial volume, normal bleed rate, CSF rate, systemic venous pressure, effectiveness of autoregulation, and

smoothing factors that affect the moving averages on pressure changes

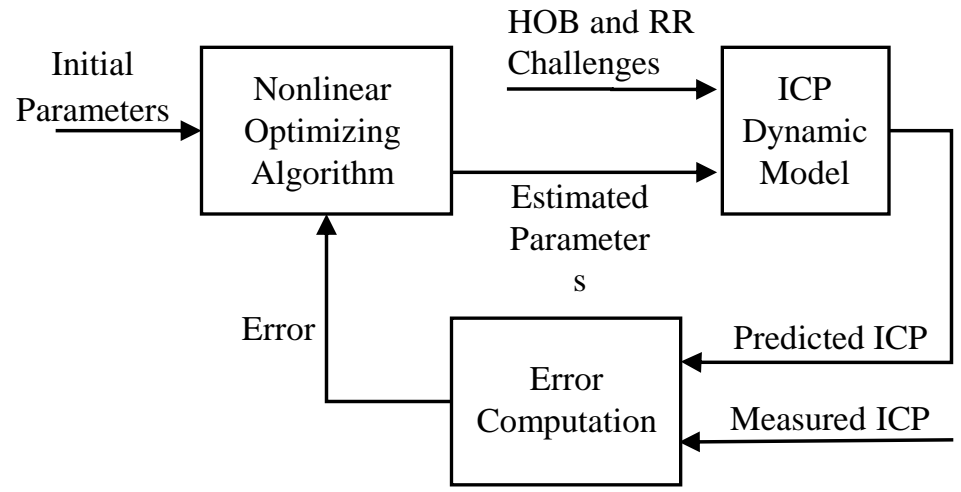

Figure 1: The Process for Estimating Patient-Specific Parameters

\section{Results}

Table 1: Summary of results
\begin{tabular}{|l|l|l|l|}
\hline $\begin{array}{l}\text { Patient and } \\
\text { Session }\end{array}$ & $\begin{array}{l}\text { Session Length } \\
\text { (minutes) }\end{array}$ & $\begin{array}{l}\text { Mean Absolute } \\
\text { Error (mmHg) }\end{array}$ & $\begin{array}{l}\text { Types of } \\
\text { Challenges }\end{array}$ \\
\hline P1, S1.5 & 12 & 0.9174 & 1 HOB \\
\hline P1, S3 & 18 & 0.8935 & 1 HOB \\
\hline P1, S4 & 45 & 3.7827 & 1 HOB, 3 RR \\
\hline P1, S5b & 35 & 3.2661 & 4 HOB \\
\hline P2, S1 & 55 & 0.6864 & 4 HOB \\
\hline P2, S4 & 65 & 0.8029 & 2 RR \\
\hline P2, S7a & 55 & 2.5520 & 5 HOB \\
\hline P2, S7b & 55 & 1.4257 & 4 RR \\
\hline P2, S9b & 55 & 0.5029 & 4 HOB \\
\hline P3, S3b & 70 & 3.0410 & 6 HOB \\
\hline P201, S2 & 50 & 1.2125 & 3 HOB \\
\hline
\end{tabular}

\begin{tabular}{|l|l|l|l|l|l|}
\hline & $\begin{array}{l}\text { All } \\
\text { sessions }\end{array}$ & $\begin{array}{l}\text { HOB } \\
\text { challenges }\end{array}$ & $\begin{array}{l}\text { RR } \\
\text { challenges }\end{array}$ & $\begin{array}{l}1-3 \\
\text { challenges }\end{array}$ & $\begin{array}{l}4-6 \\
\text { challenges }\end{array}$ \\
\hline$\mu$ & 1.73 & 1.63 & 2.00 & 0.96 & 2.18 \\
\hline$\sigma$ & 1.19 & 1.13 & 1.57 & 0.18 & 1.31 \\
\hline
\end{tabular}

Table 2: Means and Standard Deviations of Results

Conclusions

References

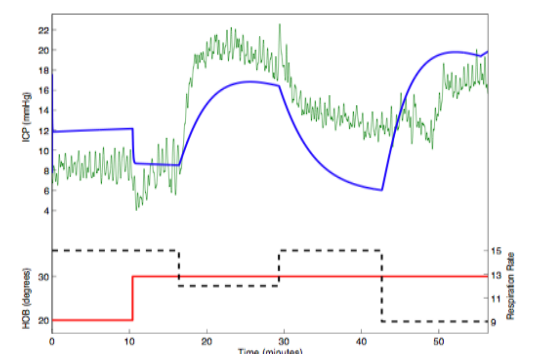

Figure 2: Patient 1, Session 4

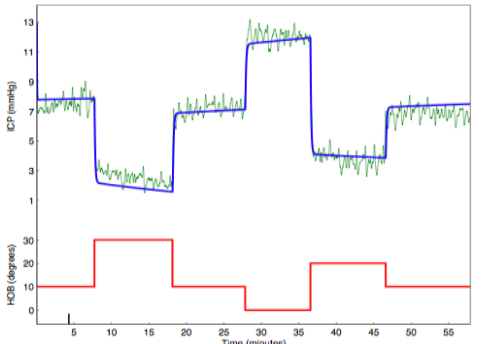

Figure 4: Patient 2, Session 9

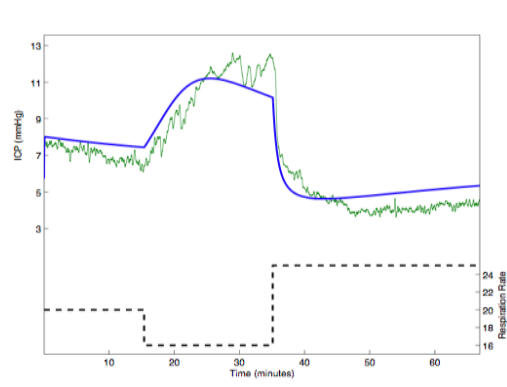

Figure 3: Patient 2, Session 4

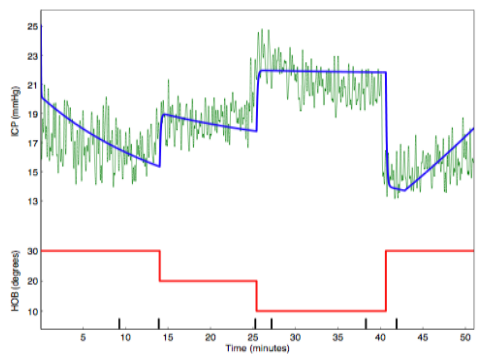

Figure 5: Patient 201, Session 2

While the model is not yet ready for clinical use, its performance continues to improve. We believe that these results support the pursuit of subject-

specific models based on clinically annotated data.

[1] Wakeland, Wayne, Joe Fusion and Brahm Goldstein. 2005. Estimation of subject specific ICP dynamic models using prospective clinical data. Biomedicine 2005 (07-09Sep2005).

[2] Ursino, M., Ter Minassian, A. Lodi, C A. Beydon, L. Cerebral hemodynamics during arterial and $\mathrm{CO}(2)$ pressure changes: in vivo prediction by a mathematical model. Am J Physiol Heart Circ Physiol. 279(5):H2439-55, 2000 Nov.

Oregon Health \& Science Univ. Complex Systems Laboratory

\section{PORTLAND STATE UNIVERSITY}

Systems Ph.D. Program

\section{Biomedical Signal Processing Laboratory} bsp.pdx.edu $\longrightarrow$ 